\title{
LAS REPRESENTACIONES ZOOMORFICAS DEL ARTE MUEBLE EN SOPORTE LÍTICO EN LA TRANSICIÓN MSF-AZILIENSE DEL NORTE PENINSULAR
}

\author{
Cristina de Juana Ortín ${ }^{1}$
}

\section{Resumen:}

Este trabajo se fundamenta en el estudio de la fauna que aparece representada en la transición MS-Aziliense, tras la catalogación de 61 piezas de arte mueble en soporte lítico pertenecientes a 17 yacimientos del norte peninsular. Se ha comprobado que dichas representaciones concuerdan con las pautas de elección intencionada de temas, al igual que ocurre con otros soportes.

Los resultados obtenidos apuntan a lo que ya señalaran Balbín y Alcolea (2003 y 2004) sobre la dificultad de utilizar las representaciones zoomórficas en el arte prehistórico para hablar de cambios climáticos. A pesar de esto, si bien no resultan infalibles a la hora de señalar características medioambientales precisas, las grafías que conviven junto a los animales en estos soportes se convierten en un elemento destacado para apoyar la continuidad en el tiempo de una tradición cultural.

\section{Palabras clave:}

fauna, arte prehistórico, estilo "V"

\section{Abstract:}

This work is based on the study of the fauna depicted in the transition MS-Azilian, after cataloging 61 pieces of furniture lithic art in support from 17 sites in the northern peninsular. It has been found that such representations match patterns deliberate choice of topics, as happens with other supports.

The results point to what already point out Balbín and Alcolea (2005) on the difficulty of using the zoomorphic representations in prehistoric art to talk about climate change. Despite this, although not infallible when pointing precise environmental characteristics, the prehistoric art coexist with the animals in these media become an important element to support the continuity in time of a cultural tradition.

\section{key words:}

fauna, prehistoric art, style " $\mathrm{V}$ "

1 cristina.juana@uah.es 


\section{Introducción}

La Universidad Rey Juan Carlos I ha tenido la iniciativa de llevar a cabo un I Congreso Internacional sobre otras arqueologías, COTARQ, al que esperamos sigan otros muchos, que recoge trabajos tanto de profesionales consolidados como de investigadores noveles dedicados a lo que se ha llamado "otras arqueologías".

Desde la organización del mismo se ha pretendido agrupar trabajos que si bien constituyen eslabones fundamentales dentro de nuestra ciencia, lo cierto es que dentro de los planes de estudio o en otros congresos parecen no tener una presencia real, siendo objeto de congresos, seminarios o jornadas que resultan del todo especificas y selectivas. Como muestra diremos que el trabajo que sigue, fundamentado en estudios de Arte Prehistórico, fue el único presentado con esta temática en dicho Congreso.

Nuestra ponencia, en la que se aborda el estudio de las representaciones animales en el arte mueble de soporte lítico, fue incluida en la sesión de arqueobiología y ha resultado posible gracias a la concesión de una Ayuda para la Formación del Profesorado Universitario del Programa Propio de la Universidad de Alcalá, de la convocatoria de 18 de mayo de 2011.

Pretendíamos con este trabajo poner en evidencia que el arte prehistórico debe ser tenido en cuenta en todo momento para el conocimiento de las sociedades del pasado en su globalidad.

Balbín y Alcolea (2005) ya insistieron en que era peligroso utilizar los animales aislados de las representaciones para hablar de situaciones de frio o de calor. En su estudio sobre la fauna representada en el arte rupestre paleolítico del interior peninsular, se ponía de manifiesto que las representaciones artísticas durante el tardiglaciar cambiaban por razones culturales y no propiamente climáticas, hecho que nosotros venimos a apoyar con las evidencias en nuestras piezas de estudio.

Al hilo de estos argumentos, como mostraremos, los animales han convivido a lo largo del Paleolítico, en mayor o menos medida, ocupando los mismos espacios y soportes con otro tipo de grafías, los signos, que nos hablan de una tradición cultural que se mantiene en el tiempo. Estos signos, constituidos por puntos y rayas, tanto pintados como grabados, adquieren una presencia destacada durante el Aziliense viniendo a formar parte del Ilamado Estilo $V$ dentro del arte prehistórico.

\section{Objetivos}

El principal objetivo de este trabajo es prestar una atención preliminar a las representaciones de animales versus representaciones de signos en el arte mueble sobre soporte lítico que, en la transición del MSF-Aziliense del norte peninsular, 
conviven con una paulatina disminución en número de las estaciones de arte rupestre.

El motivo principal para detenernos en este tipo de soportes es porque dentro del ámbito de "lo mueble" éstas comparten su misma naturaleza con las representaciones parietales, y, además, como ocurre en aquellas, la mayoría de las veces dichos soportes no son modificados en modo alguno.

Estas características nos invitan a preguntarnos en dos direcciones: por un lado, si la fauna representada en soportes líticos muebles es indicativa de algún cambio paleoambiental. Y, por otro lado, hasta que punto, estos soportes son indicativos de la existencia de continuidad en lo conceptual y simbólico con representaciones anteriores, viniendo a conformar el Estilo V.

\section{Consideraciones previas}

Respecto a la fauna representada. A principios de los noventa, Sauvet y Wlodarczyk (1995:193-211) llevaron a cabo una clasificación de especies por frecuencia de aparición en las representaciones parietales paleolíticas expresado en porcentajes que resultó así:

\begin{tabular}{|l|l|l|l|}
\hline Caballo & 28.7 & Reno & 3.7 \\
\hline Bisonte & 22.2 & Oso & 1.4 \\
\hline Cabra & 9.5 & León & 1.2 \\
\hline Uro & 6.1 & Rinoceronte & 0.5 \\
\hline Ciervo & 5.8 & Pez & 1.0 \\
\hline Cierva & 7.3 & Humano & 3.5 \\
\hline Mamut & 7.8 & Diversos & 1.3 \\
\hline
\end{tabular}

Fig.1. Porcentajes de aparición de especies en representaciones rupestres según Sauvet y Wlodarczyk.

Viendo estos resultados comprobamos como tan sólo con el caballo y el bisonte tendríamos el $50 \%$ de las representaciones, lo que verifica una selección del todo intencionada. Se pueden apreciar de forma evidente cuatro grupos de frecuente aparición: équidos, bóvidos, cérvidos y cápridos, complementados por otras especies como el mamut, el rinoceronte y otros cuya representación es menor y su dispersión geográfica más irregular (Alcolea y Balbín, 2005:518-519; Sauvet, 1993). 
Respecto a la elección de soporte. La primera gran obra de referencia que recogía el arte mueble del norte peninsular fue Arte mueble del Paleolítico cantábrico (Barandiarán 1972). En dicha obra las piezas sobre soporte lítico constituyen tan sólo 9,1\% del total, de las cuales, la mayor parte pertenecen al yacimiento de La Paloma. El grueso de las piezas recogidas había sido realizado sobre hueso. Es posible que esta diferencia tan grande en la elección de los soportes no se corresponda con la realidad de aquel tiempo, y se debiera a la falta de interés en las propias excavaciones por cantos o piezas líticas de pequeño tamaño que no presentaban signos de haber estado implicadas en los procesos de talla.

Aún así en excavaciones Ilevadas a cabo con metodología actual y estrictamente rigurosa, estas diferencias en la elección del soporte siguen siendo llamativas, en ocasiones por el motivo contrario, como en Estebanvela donde prevalece el soporte pétreo frente al hueso (García Díez 2013:499).

Aunque por estos motivos no podemos valorar la implicación en la elección del soporte, ni tampoco es objetivo de estas líneas, lo que sí sabemos es que las piezas de arte mueble sobre soporte lítico según Barandiarán (1972:248) se caracterizan por:

- La piedra empleada casi siempre no está acondicionada

- Las calidades de resistencia y compacidad de los soportes influyen en las técnicas de grabado. De esta forma los trazos incisos son finos y de huella estrecha en los soportes líticos de grano menudo y carácter compacto (algunos esquistos, cuarcitas y calizas duras), y son grabados profundos y planos los realizados sobre cantos y placas de grano grueso.

Además, debemos indicar que la pintura en los soportes líticos también está presente y que adquiere un mayor protagonismo a medida que avanzamos en el tiempo.

Respecto a la continuidad de los temas. La valoración de los soportes muebles decorados permite apreciar cierta continuidad en el tiempo en lo referente a las manifestaciones artísticas, hecho que llevó a Breuil (1974: 405) a proponer "la primera explicación teórica estilística para la transición gráfica paleolíticopospaleolítico hablando en más de una ocasión de una "quinta fase" del Arte Paleolítico contemporánea al Aziliense" (Bueno, Balbín y Alcolea, 2008: 258259) que completaría la secuencia de estilos que elaboró Leroi-Gourhan.

En las obras Les religions de la préhistoire (1964), Le geste et la parole (1964b) y, sobre todo, en La préhistoire de l'art occidental (1965) Leroi-Gourhan expuso su secuencia cronológica válida tanto para el arte parietal como para el arte mobiliar. 
Leroi-Gourhan para su propuesta de secuencia de estilos utilizó precisamente el arte mueble y los fragmentos de pared decorados, que habían aparecido en estratigrafía al desprenderse, lo que le permitió elaborar una propuesta basada en contexto arqueológico. Dentro de los estilos de Leroi-Gourhan, el estilo IV se caracterizaría porque las figuras buscan el realismo más allá del naturalismo que había presidido las representaciones anteriores, lo cual supondría el final del arte paleolítico.

Durante el Epipaleolítico cantábrico (11000-5500 B.P.) las representaciones figurativas van a ir desapareciendo paulatinamente, encontrándose un arte esquemático menos abundante pero que mantiene continuidad con motivos esquemáticos del Magdaleniense Superior (González Sainz y González Morales, 1986:260)

Los hallazgos en el sur de Cataluña y en el norte de la Comunidad Valenciana como son los conjuntos de Moli del Salt (García Díez y Vaquero, 2006), Parpalló (Villaverde, 2004), Matutano (Olaria, 2008) o Tossal de la Roca (Ripoll y Cacho, 1987) citados por Martínez Moreno, Villaverde y Mora Torcal (2011:159-160) vienen a confirmar esta continuidad entre el arte del MSF y el del Epipaleolítico.

De igual modo, los 43 soportes decorados hallados en el yacimiento de La Peña de Estebanvela han servido, debido a su repartición en tres conjuntos sedimentarios diferentes, "para caracterizar la variabilidad interna existente durante el Magdaleniense final (niveles I y II correspondientes a ocupaciones humanas acontecidas entre hace 11.770 y 10.580 años BP) - lapso de entre 13.800 y 12.570 años BP calibrados al 95\%-)" (García Díez 2013:503).

Hallazgos como los de Fariseu en Portugal o de Estebanvela (Segovia) en España, junto con otras evidencias, han servido a Bueno, Balbín y Alcolea (2007 y 2008:256) para afirmar que existen "interesantes elementos de reflexión para una continuidad Paleolítico-Epipaleolítico" que vendría a corroborar la hipótesis de Breuil.

El estilo $V$ está presente en cuevas, en grabados al aire libre y en arte mueble con buenas referencias de cronologías absolutas de entre el 11500BP y el 9000BP que avalan la continuidad del Arte Paleolítico en todo el sur de Europa (Bueno, Balbín y Alcolea 2007, 2008:257-261).

Las representaciones adscritas al "estilo V", encuadradas dentro del ámbito Epimagdaleniense/Epipaleolítico correspondiente al Final del Tardiglaciar se caracterizan principalmente porque:

- Los temas figurativos conviven con lo abstracto

- Hunde sus raíces estilísticas y temáticas en el MSF, con trazos simples y sencillos aparentemente sin organización, aspas o reticulados son conocidos en contextos crono-culturales anteriores. 
- Temas animales: équidos, bóvidos y cérvidos

- Representaciones más pequeñas

- Destaca la utilización de trazos repetidos y superpuestos, así como las formas triangulares.

\begin{tabular}{|c|c|c|}
\hline Imagen & $\begin{array}{l}\text { Adscripción } \\
\text { Cultural }\end{array}$ & Descripción \\
\hline Tito Bustillo & $\begin{array}{l}\text { Magdaleniense } \\
\text { Superior }\end{array}$ & $\begin{array}{l}\text { Fragmento de plaquita de piedra grabada a } \\
\text { trazo fino con el esbozoo de la cubeza de um } \\
\text { animal, quizá un caballo, y cruzándolo } \\
\text { trasversalmente algunos trazos lineales que } \\
\text { posiblemente conforman un tema de } \\
\text { triangulos MOURE, 1975; CORCHON, } \\
\text { 1986. }\end{array}$ \\
\hline Tito Bustillo & $\begin{array}{l}\text { Magdaleniense } \\
\text { Superior }\end{array}$ & $\begin{array}{l}\text { Colgante de forma ovalada y sección } \\
\text { aplanada, roto por la perforación, y } \\
\text { decorado con marcas en el contorno y con } \\
\text { puntuaciones, cuatro hileras por cada cara } \\
\text { y alternado } 5 \text { y } 6 \text { puntos en la superior, y } \\
\text { un número no precisado por roturas en la } \\
\text { opuesta. MOURE, 1976, CORCHÓN, } \\
\text { 1986. }\end{array}$ \\
\hline La Paloma & $\begin{array}{l}\text { Magdaleniense } \\
\text { Superior Final }\end{array}$ & $\begin{array}{l}\text { Placa en pizarra grabada en una cara con } \\
\text { incisjones rectilineas cruzadas de forma } \\
\text { irregular. CORCHÓN, 1971, } 1986 \text {. }\end{array}$ \\
\hline Balmori & Aziliense & $\begin{array}{l}\text { Se han grabado dos trazos transversales, } \\
\text { rellenos por otros dos menores oblicuos, } \\
\text { parcialmente interrumpidos por otros dos } \\
\text { apendiculares, todo lo cual sugiere la } \\
\text { silueta de un animal indeterminado, } \\
\text { probablemente incompleto (sin cabeza). } \\
\text { OBERMAIER, 1921; CORCHÓN, 1986 }\end{array}$ \\
\hline Los Azules & Aziliense & $\begin{array}{l}\text { Canto prooedente de una sepultura. } \\
\text { Presenta en la eara superior un punto de } \\
\text { color de negro y una franja coloreada } \\
\text { semicircular. En una cura lateral se sitúan, } \\
\text { asimismo, dos puntos en negro y una } \\
\text { pequena mancha. FERNÁNDEZ- } \\
\text { TRESGUERRES, 1982; CORCHÓN, } \\
\text { 1986. }\end{array}$ \\
\hline
\end{tabular}

Fig.2. Recogemos en esta tabla algunos ejemplos dónde se observan que los puntos y rayas, grabados o pintados se mantienen en el tiempo en soportes de similares características.

\section{Metodología y resultados}

Para este trabajo hemos analizado 61 piezas muebles en soporte lítico, adscritas al Magdaleniense Superior y el Aziliense, pertenecientes a un total de 17 yacimientos que recorren toda la cornisa cantábrica, desde Asturias al P. Vasco. Dichos yacimientos son: Tito Bustillo, El Castillo, La Paloma, Collubil, El Pendo, El Valle, Atxeta, Santimamiñe, Lumentxa, Urtiaga, Aitzbitarteiu, Cueva Oscura de Ania, Los Azules, La Riera, Balmori, El Pindal y Urratxa. 
Las piezas en cuestión son las recogidas en las obras de referencia anteriormente citadas (Barandiarán (1972) y Corchón (1986)) y en los casos en los que nos ha sido posible, las que hemos consultado personalmente.

La información pormenorizada de cada una de las piezas analizadas ha sido recogida en fichas elaboradas en File Maker siguiendo la metodología que ya propusiéramos (Juana, 2014).

\begin{tabular}{|c|c|c|c|c|c|c|c|c|c|c|c|c|}
\hline \multirow[t]{2}{*}{ YACIMIENTO } & \multicolumn{6}{|c|}{ Magdaleniense } & \multicolumn{5}{|c|}{$\overline{\text { Aziliense }}$} & \multirow[t]{2}{*}{ Total Piezas } \\
\hline & cer & cap & eq & & otro & sig & cer| & сар 6 & eq bo & otro & sig & \\
\hline Tito Bustillo & 3 & & \begin{tabular}{|l|}
4 \\
\end{tabular} & 1 & & \begin{tabular}{l|l}
3 \\
\end{tabular} & & & & & & 11 \\
\hline El Castillo & 1 & & \begin{tabular}{|l|}
1 \\
\end{tabular} & & & & & & & & & 2 \\
\hline La Paloma & & & & & $\overline{1}$ & 7 & & & & & & 8 \\
\hline Collubil & & & & & $\overline{1}$ & & & & & & & 1 \\
\hline EPendo & & & & & & 3 & & & & & & 3 \\
\hline El Valle & & & & & & & & & & & 2 & 2 \\
\hline Atxeta & & & & & 2 & 1 & & & & & & 3 \\
\hline Santimamiñe & & & & & 1 & & & & & & & 1 \\
\hline Lumentxa & & & \begin{tabular}{|l|}
1 \\
\end{tabular} & & & & & & & & & 1 \\
\hline Urtiaga & & 1 & \begin{tabular}{|l|}
1 \\
\end{tabular} & & 3 & & & & & & & 5 \\
\hline Aiztbitartelu & 1 & & & & & & & & & & & 1 \\
\hline $\begin{array}{c}\text { Cueva } \\
\text { Oscura A. }\end{array}$ & & & & & & & & & & & 3 & 3 \\
\hline Los Azules & & & & & & & & & & & 15 & 15 \\
\hline La Riera & & & & & & & & & & & 1 & 1 \\
\hline Balmori & & & & & & & & & & 1 & 1 & 2 \\
\hline Pindal & & & & & & & & & & & 1 & 1 \\
\hline Urratxa & & & & & & & & & & & 1 & $\overline{1}$ \\
\hline & 5 & 1 & \begin{tabular}{|l|}
7 \\
\end{tabular} & 1 & 8 & \begin{tabular}{|l|l|}
14 & \\
\end{tabular} & & & & 1 & 24 & 61 \\
\hline
\end{tabular}

Fig.3. Relación de animales y signos en soportes muebles de tipo lítico de cada yacimiento. En el grupo de otros se engloban representaciones de animales puntuales como los carnívoros, o de cuadrúpedos no identificables. Tampoco se han tenido en cuenta la asociación de más de un animal, sólo aquellos bien identificados.

En el área cantábrica, Leroi-Gourhan (1965:438) observo una divergencia en la temática entre el arte parietal y el mueble, de manera que mientras en lo parietal predomina la asociación caballo-bisonte, en lo mueble éstas son relativamente escasas, debiendo subrayarse casi la total ausencia de bisontes en el repertorio iconográfico mueble (Barandiarán 1972:267). Este hecho puede constatarse en nuestro estudio, que sólo tiene en cuenta las piezas 
muebles en soporte lítico, donde los cérvidos toman total protagonismo en el Magdaleniense Superior.

Como observamos, durante el MS las especies más representadas son los cérvidos y équidos, mientras que con gran diferencia en el Aziliense lo son los signos, que ya estaban presentes en periodos anteriores.

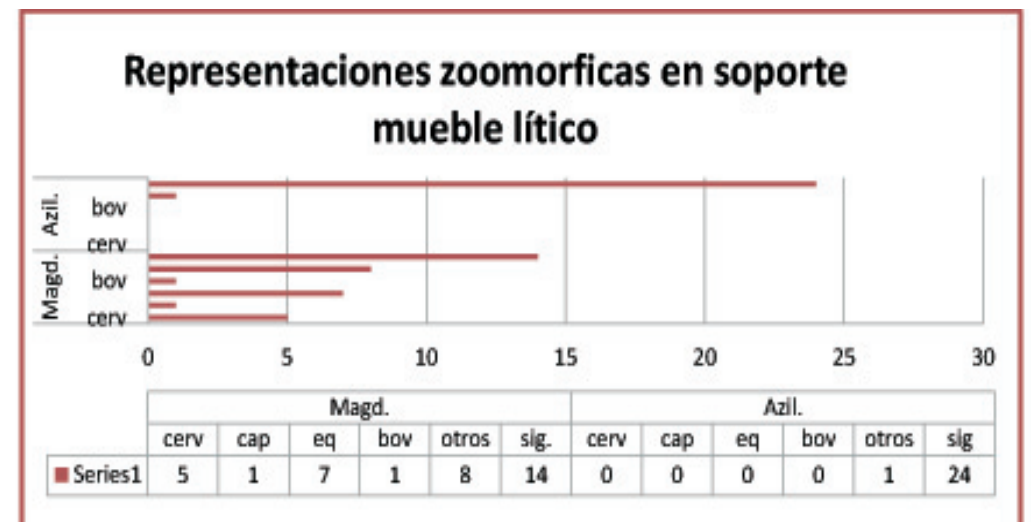

Fig.4. Total de representaciones zoomórficas y de signos en función de la adscripción cultural.

\section{Consideraciones finales}

Respecto a las grafías. Hemos verificado que las piezas de arte mueble en soporte lítico se ajustan perfectamente a las descripciones de conjunto sobre arte mueble cantábrico.

Las representaciones de los animales, al final del Magdaleniense en el área cantábrica se caracterizan por resultar más estilizadas que en momentos anteriores, sobre todo en lo referente al sujeto estilizado más típico, la cabra, se dan situaciones de representaciones dobles y se conocen algunos ejemplos de incluso tres y cinco individuos. Otra característica del final del Magdaleniense cantábrico es que se hacen frecuentes las representaciones parciales de los sujetos, aunque se percibe un renovado interés por la expresión plástica del volumen mediante la escultura, modelado o relieve, algo que ha heredado del Magdaleniense Medio (Corchón 1986:104-105).

Al llegar el Aziliense, a comienzos del Epipaleolítico Cantábrico, las representaciones animales parecen desvanecerse, convirtiéndose en protagonistas absolutos de las representaciones muebles los signos, en forma de puntos o bandas mayoritariamente (Los Azules, El Valle, El Pindal), aunque éstos en ningún caso son originales, sino una prolongación de algunos de los motivos del Magdaleniense superior final (Corchón 1986:105). 
Respecto a los indicadores climáticos o paisajísticos. Atendiendo a las preguntas que nos hacíamos inicialmente, hay que tener en cuenta que en el límite entre MSF y Aziliense, hacía la 13500 cal BP, observamos unas condiciones templadas propias de los momentos finales del Interestadio del Tardiglacial, que los datos malacológicos y palinológico muestran como una mejora climática a lo largo del Aziliense (García Moreno y Gutiérrez Zurigasti, 2012:203).

Por tanto, hubiera sido significativo que encontrásemos representadas especies de fauna fría (F.F), ya que las características que definen tradicionalmente al término "mammouth steppe" no pueden ser aplicadas al menos, en el caso de la PI. Estas especies de F.F Ilegan a la PI sólo durante periodos temporales específicos y durante unas condiciones climáticas características. De esta forma el mamut, el rinoceronte lanudo y el antílope saiga aparecen de forma puntual en la PI durante el Tardiglacial en dos fases claramente definidas, la primera entre ca. 20700 y 17500 BC cal (Álvarez y Jöris, 2009:67), que son anteriores a nuestro periodo de estudio.

Dicho esto, y observando que los principales animales representados son ciervos (Cervus elaphus) y équidos (Equus caballus) que son especies euritermas, debemos tener la precaución de entender, que una especie euriterma es aquella que se adapta a medios ambientes diferentes y no obligadamente aquella perteneciente a biotopos de clima moderado. La información que podemos obtener, por tanto, de dichas especies, más que en relación al clima estaría relacionada con las características del paisaje en el que se desarrollan. Los caballos son una especie que se adapta mejor a espacios abiertos y los ciervos prefieren zonas arboladas (Alcolea y Balbín, 2005:519).

Recordemos que durante el desarrollo del Tardiglacial los niveles marinos ascendieron casi modo constante, produciéndose una profunda modificación de los paisajes litorales. Este hecho es de gran relevancia a la hora de valorar en conjunto a la fauna regional y los sistemas de explotación. Los caballos se vieron privados de un hábitat adecuado debido al avance de los bosques y los ciervos, aunque son la especie dominante en la mayoría de los yacimientos a lo largo del Paleolítico Superior, pensamos que su presencia debió verse reducida pues, los bosques cerrados caducifolios, no debían resultarles muy favorables a su modo de vida (González Sainz y González Morales, 1986:260).

Respecto al valor conceptual y simbólico. Balbín y Alcolea (1994:128$129 ; 2005: 520)$ han insistido en que las representaciones de animales en el Paleolítico son formas que han extraido de su realidad más próxima pero que no la reflejan de forma mecánica ni exacta. Los animales que observamos conforman un código cultural que expresan múltiples realidades (Balbín y Alcolea, 1999:45; 2005:520). Este fenómeno como apuntan Alcolea y Balbín (2005:520) tiene su máxima evidencia en Lascaux (Bouchud, 1979:148) donde 
la fauna representada y la presente en el registro arqueológico apenas tiene coincidencia.

En las tablas que aportamos las representaciones tienen una clara dualidad, la de los zoomorfos y la de los signos. Los signos, aquellas representaciones no figurativas que no podemos identificar, parecen contener un significado muy concreto que permanece en el tiempo. Este tipo de decoración de líneas finas con puntos o pequeños trazos adosados según González Sainz (1986:255) encuentran buenos paralelos con piezas mobiliares del Aziliense francés y del romanelliense italiano.

Por el contrario, los animales, parecen adquirir un sentido simbólico estricto pues llegan a prácticamente desparecer en este tipo de soportes cantábricos. No ocurre lo mismo en otras regiones geográficas para las mismas cronologías como en Estebanvela, donde hay soportes líticos con caballos o Fariseu, con ciervos, en estos casos representaciones faunísticas bajo claras características del aludido Estilo $V$, y que en cualquier caso son muestra de un cambio de mentalidad y entorno cultural que es paralelo a los cambios climáticos y de los modos de subsistencia.

Por último, y como ya señalara E. Corona-M. (2010), creemos que es necesario volver a incluir en la investigación arqueobiológica el proceso de estudio de las representaciones zoomorfas. Más allá de la identificación de las propias especies que pueden aportarnos cierta información en lo relativo a la morfología, distribución y ecología, al relacionarlos con los aspectos culturales, como es el arte prehistórico, o mejor dicho, el estudio de las grafías prehistóricas, podremos obtener información acerca de su valor simbólico, relevancia cultural o geográfica, así como su asociación a determinados soportes.

\section{Bibliografía}

ALCOLEA GONZÁLEZ, J. J., \& DE BALBíN BEHRMANN, R. (2003). Témoins du froid. La faune dans I'art rupestre paléolithique de l'intérieur péninsulaire. L'Anthropologie, 107(4), 471-500.

ALCOLEA GONZÁLEZ, J. J. A., \& DE BALBÍN BEHRMANN, R.,2003. Testigos del frio: la fauna en el arte rupestre paleolítico del interior peninsular. In Geoarqueología y Conservación del patrimonio (pp. 517-536).

ÀlVAREZ FERNÁNDEZ, E. Y JÖRIS, O., 2009. El significado cronológico de algunas especies de faunia fría durante el Tardiglaciar en la Península Ibérica. Zephyrvs, vol. 51, pp. 61-86.

BALBÍN BEHRMANN, R., GONZÁLEZ, J. J. A. Y SANTONJA, M., DE, 1994, Siega Verde y el arte rupestre paleolítico al aire libre. In 6 Coloquio Hispano-Ruso de Historia (pp. 5-19). Fundación Cultural Banesto.

BALBIN BEHRMANN, R., \& ALCOLEA, J. J.,1999, Vie quotidienne et vie religieuse. Les sanctuaires dans I'Art Paléolithique. L'Anthropologie, 103, 23-49. 
BARANDIARÁN, I., 1972, Arte mueble del paleolítico cantábrico. Zaragoza: Departamentos de Prehistoria y Arqueología de la Universidad.

BOUCHUD, J.,1979, La faune de la grotte de Lascaux in Lascaux inconnu. Supplément à Gallia Préhistoire Paris, 12, 147-152.

BREUIL, H., 1974. Quatre cents siêcles d'Art Pariétal. París: Editions Max Fourny.

BUENO RAMÍREZ, P. AND BALBÍN BEHRMANN, R. Y ALCOLEA GONZÁLEZ, J.J.,2008, Estilo $V$ en el ámbito del Duero: cazadores finiglaciares en Siega Verde (Salamanca) Arte prehistórico al aire libre en el sur de Europa, 2008.

CORCHÓN, M.S., 1986. El arte paleolítico cantábrico contexto y análisis interno. 1a ed. Madrid: Ministerio de Cultura ISBN 8450567882.

CORONA-M., E., 2010. El arte rupestre, expresión de las relaciones entre hombre y fauna. El Tlacuache 410, pp. 3.

GARCÍA DíEZ, M., 2013. La expresión gráfica de La Peña de Estebanvela (Segovia) en el contexto de los últimos grupos cazadores-recolectores europeos. In: Ocupaciones magdalenienses en el interior de la Península Ibérica. La Peña de Estebanvela (Ayllón, Segovia) Carmen Cacho, Juan Antonio Martos y Jesús Valdivia, 2013 ed. Junta de Castilla y León. CSIC., pp. 471-514.

GARCÍA DÍEZ, M. Y VAQUERO, M., 2006. La variabilité graphique du Molí del Salt (Vimbodí, Catalogne, Espagne) et I'art mobilier de la fin du Paléolithique supérieur à l'est de la Péninsule Ibérique. L'Anthropologie, vol. 110, no. 4, pp. 453-481.

GARCÍA MORENO, A. Y GUTIÉRREZ ZUGASTI, F. I., 2012. Cambios climáticos al final del Tardiglacial: una aproximación multidisciplinar a las evidencias paleoclimáticas del valle de Asón (Cantabria) El Paleolítico Superior Cantábrico: actas de la Primera Mesa Redonda, San Román de Candamo (Asturias), 26-28 de abril de 2007.

GONZALEZ SAINZ, C., 1986, La prehistoria en Cantabria (Vol. 1). Ediciones Tantin.

JUANA, C. d., 2014. Inicio al estudio de las piezas muebles finipaleolíticas y azilienses sobre soporte lítico. A.R.P.I. Arqueología y Prehistoria Del Interior Peninsular, vol. 01, pp. 47-63.

LEROI-GOURHAN, A., 1964. Le geste et la parole, 2 vols. Albin Michel, Paris, vol. 1.

LEROI-GOURHAN, A., 1964b, Les religions de la préhistoire.

LeROI-GOURHAN, A., Coppens, Y., Delluc, G. y Delluc, B., 1965. Préhistoire de I'art occidental. L. Mazenod.

MARTíNEZ-MORENO, J., VILLAVERDE, V. Y MORA TORCAL, R., 2011. La placa grabada de Balma Guilanyà (Prepirineo de Lleida) y las manifestaciones artísticas del Mesolítico de la Península Ibérica. Trabajos De Prehistoria, vol. 68, no. 1, pp. 159-173. 
OLARIA, C., 2008. Grafismo mobiliar magdaleniense de Cova Matutano (Vilafamés, Castellón) en el contexto del Mediterráneo peninsular. Monografies de Prehistòria i Arqueologia Castellonenques 7. ed. Castellón: Diputació de Castelló.

RIPOLL, S. Y CACHO, C., 1987. Art mobilier du Paléolithique méditerranéen espagnol. Quelques nouvelles techniques. J. Clottes (Ed.): L'art Des Objets Au Paléolithique 1 - L'art Mobillier Et Son Contexte. Actes Du Colloque De FoixLe Mas d'Azil, pp. 287-293.

SAUVET, G., 1993, Thématique de lárt parietal. Introduction. Le problème de la détermination. En "L'Art Pariétal Paléolithique. Techniques et métodes d’étude. Documents Préhistoriques, 5. París. Pp 83-86.

SAUVET, G. Y WLODARCZYK, A., 1995. Eléments d'une grammaire formelle de I'art pariétal paléolithique. L'anthropologie, vol. 99, no. 2-3, pp. 193-211.

VILLAVERDE, V., 2004. Arte mueble paleolítico en el Mediterráneo occidental: contexto y diversidad regional. La Materia Del Lenguaje Prehistórico.El Arte Mueble Paleolítico De Cantabria En Su Contexto.Instituto Internacional De Investigaciones Prehistóricas De Cantabria.Santander, pp. 67-84. 